\title{
Electronic Infectious Disease Surveillance System during Humanitarian Crises in Yemen
}

\author{
Kamran Ahmed*, Mohammad Dauod Altaf and Fekri Dureab \\ World Health Organization, Sana'a, Yemen
}

\section{Objective}

The intended objective of the Electronic Disease Early Warning System (eDEWS) is to minimize morbidity and mortality due to communicable diseases through detection of potential outbreaks at their earliest possible stage using a novel modeling approach that mainly facilitates the transformation of data into actionable information.

\section{Introduction}

Effective communicable disease control through rapid detection and prompt response to outbreaks is one of the priorities during a humanitarian crisis, as communicable diseases can be a major cause of morbidity and mortality in emergencies, particularly in countries with poor disease surveillance mechanisms. Yemen is among many developing countries being hit by conflicts, displacement of population and disruption of basic services, where among other public health risks and threats the population is exposed to risks of several communicable diseases. Thus, effective preventive and control measures through early detection and rapid identification of infectious diseases and provision of a public health response to communicable disease outbreaks, a crucial priority health intervention, need to be directed towards diseases that are endemic and particularly those which can potentially cause excess numbers of mortality and morbidity within a short span of time.

\section{Methods}

The eDEWS project was introduced in March 2013 for outbreak detection include immediate alert reporting and weekly electronic data collection on several syndromes from about 100 health facilities in 4 governorates in Yemen on pilot basis. The eDEWS platform was developed to capture data electronically on prioritized epidemic prone diseases on real time basis (information is delivered immediately after collection to the district or governorate hubs for validation) using mobile (GPRS) based \& web (internet) based interfaces. It also facilitates secure automatic electronic transmission and analysis of data and dissemination of information to main stake holders. Alert SMS notifications are automatically generated for immediate response, based on defined criteria of surpassing median endemic index. Only verified SMS alert notifications are considered as true alerts.

\section{Results}

As of 30 August 2013, the eDEWS detected 745 alerts and 4 outbreaks in 100 selected sentinel sites in 4 pilot governorates, mainly of suspected pertussis, measles, acute viral hepatitis and dengue. Alert investigation appeared well structured and timely, but problems with laboratory confirmation were evident. Overall percent reporting rates of eDEWS notifiable diseases remained $>70 \%$ with steady increase to $>95 \%$ in few months. eDEWS central database generated 906 SMS alerts, of which 745 SMS alerts were verified as true alerts and more than $85 \%$ of true alerts were investigated and responded within 24 hours. Of these 730 true alerts (positive predictive value [PPV] 82. $2 \%$ ), or about 30 true alerts per week, 4 were confirmed as outbreaks. Prompt public response proved to be effective and efficient with early containment of detected outbreaks.

\section{Conclusions}

Despite the many challenges, the results of pilot project indicate that eDEWS can successfully fill the gaps of the current routine disease surveillance system in the rapid detection of potential outbreaks, in the effective monitoring of communicable diseases, and in the notification of events of public health importance. The developed system also provides possibilities for replication or adaptation in other humanitarian settings for improving the overall disease surveillance capacity and capabilities, and expanding the system into a more robust electronic system for public health surveillance in the longer future.

\section{Keywords}

eDEWS; disease surveillance; emergencies; outbreaks; communicable disease control

\section{Acknowledgments}

We wish to thank Dr Abdul Hakeem Kohlani and Dr Reema (MoPHP Yemen), Dr Ghulam Popal \& Dr Ahmed Shadoul (WRs WHO), Dr Maha Obadi (FETP Yemen) and the eDEWS and other WHO staff, too numerous to list individually, who provided us with prompt help, information and facilitation. Last but not least, we extend our thanks to UNOCHA Yemen who funded the pilot phase of eDEWS project in Yemen.

\section{References}

WHO (2000) An integrated approach to communicable disease surveillance. Weekly Epidemiological Record 75, 1-8.

Watson, J. T., M. Gayer and M. A. Connolly (2007). "Epidemics after natural disasters." Emerging Infectious Diseases 13(1): 1-5.

\section{*Kamran Ahmed}

E-mail: drkamranrajput@hotmail.com 